\title{
Agentes Comunitários de Saúde e o Trabalho com Usuários de Drogas: Obstáculos e Desfechos Possíveis
}

\section{Community Health Agents and Work with Drug Users: Possible Obstacles and Outcomes \\ Agentes de Salud Comunitaria y el Trabajo con Usuarios de Drogas: Posibles Obstáculos y Resultados}

Ana Carolina Ametlla Guimarães

Secretaria Municipal de Saúde de Campo Grande/MS

Iolly Nacasato

Rayssa Nacasato

Leila Simone Foerster Merey

Universidade Federal de Mato Grosso do Sul (UFMS)

Carolina Gomes Carrilho ${ }^{1}$

Universidade Católica Dom Bosco (UCDB)

André Barciela Veras

Universidade Estadual de Mato Grosso do Sul (UEMS)

Alessandro Diogo De Carli

Mara Lisiane de Moraes dos Santos

Universidade Federal de Mato Grosso do Sul (UFMS)

\begin{abstract}
Resumo
Introdução: Na Estratégia Saúde da Família (ESF), o trabalho do Agente Comunitário de Saúde (ACS) é ponto inicial de ancoragem das ações em saúde voltadas à comunidade. Objetivo: compreender o entendimento dos ACS em relação à abordagem da temática das drogas no território em que atuam. Métodos: Estudo qualitativo, realizado com 6 ACS de uma Equipe de Unidade de ESF. Definiu-se a amostra por exaustão. Foram realizadas entrevistas, posteriormente transcritas e organizadas conforme o Discurso do Sujeito Coletivo. Resultados: Foram evidenciados entraves e equívocos no processo de trabalho dos ACS no manejo das demandas relacionadas ao uso de álcool e drogas. Conclusão: A percepção dos ACS sobre a temática do uso de drogas no cotidiano do serviço de saúde é cautelosa e restrita. Mesmo diante da identificação disto como um problema do território, suas intervenções ocorrem mediante manifestação voluntária do usuário, quando este busca o serviço de saúde.

Palavras-chave: estratégia saúde da família, agentes comunitários de saúde, álcool e/ou outras drogas
\end{abstract}

\section{Abstract}

Introduction: In the Family Health Strategy (FHS), the work of the Community Health Agent (CHA) is the starting point for anchoring health actions aimed at the community. Objective: to comprehend the understanding of the $\mathrm{CHA}$ in relation to addressing the theme of drugs in the territory in which they operate. Methods: Qualitative study, carried out with 6 CHA from an FHS Unit Team. The sample was defined by exhaustion. Interviews were carried out, later transcribed and organized according to the Collective Subject Discourse. Results: Obstacles and misunderstandings were evidenced in the work process of the $\mathrm{CHA}$ in handling the demands related to the use of alcohol and drugs. Conclusion: The perception of $\mathrm{CHAs}$ on the theme of drug use in the daily routine of the health service is cautious and restricted. Even with the identification of this as a territory problem, their interventions occur through voluntary manifestation of the user, once he seeks the health service.

Keywords: family health strategy, community health agents, alcohol and/or other drugs

\section{Resumen}

Introducción: En la Estrategia de Salud Familiar (ESF), el trabajo del Agente de Salud Comunitaria (ASC) es el punto de partida para anclar las acciones de salud dirigidas a la comunidad. Objetivo:

\footnotetext{
${ }^{1}$ Endereço de contato: Avenida Tamandaré, 6000, Campo Grande, Mato Grosso do Sul, Brasil. Código Postal: 79117-900. Telefone: (67) 99837-2727. E-mail: carolcarrilho1@hotmail.com
} 
comprender lo entendimiento de la ASC en relación con el tema de las drogas en el territorio en el que operan. Métodos: Estudio cualitativo, realizado con 6 ASC de un equipo de la Unidad ESF. La muestra se definió por agotamiento. Las entrevistas se llevaron a cabo, luego se transcribieron y se organizaron de acuerdo con el Discurso del Sujeto Colectivo. Resultados: se evidenciaron obstáculos y malentendidos en el proceso de trabajo de la ASC en el manejo de las demandas relacionadas con el uso de alcohol y drogas. Conclusión: La percepción de los ASC sobre el tema del consumo de drogas en la vida diaria del servicio de salud es cautelosa y restringida. Incluso con la identificación de esto como un problema del territorio, sus intervenciones ocurren a través de la manifestación voluntaria del usuario, cuando este busca el servicio de salud.

Palabras clave: estrategia de salud familiar, agentes comunitarios de salud, alcohol y/u otras drogas

\section{Introdução}

No Brasil, a implementação da Estratégia Saúde da Família (ESF) tem sido a principal aposta no processo de reorientação do modelo de saúde no SUS. Entretanto, esta tem se mostrado um desafio tanto para sua expansão quanto no que tange à modificação das práticas instituídas. Várias questões precisam ser debatidas, entre as quais a própria constituição do trabalho em saúde, cujos processos não têm, na prática, privilegiado o vínculo nem a noção de um trabalho em equipe (Galavote, 2011), fatores que podem limitar o alcance das ações de saúde realizadas nesse contexto.

Em um modelo de intervenção que transcende o âmbito individual e clínico, são necessárias transformações no modo de conduzir e organizar o processo de trabalho. Assim, cada profissional deve desempenhar suas atribuições em um processo de decisões coletivas, cujo produto será fruto de um trabalho que se tece com a contribuição peculiar das diversas áreas profissionais e de seus campos de conhecimento (Brasil, 2001). Dessa forma, autores como Merhy (2003) apontam que a mudança no modelo assistencial de saúde passa, necessariamente, pela reorientação do processo de trabalho em saúde, na perspectiva de se constituir como um trabalho que seja, ao mesmo tempo, coletivo e voltado para o coletivo da comunidade.

Sabemos que tanto a prevenção primária ao uso de álcool e outras drogas quanto o acompanhamento das pessoas que já apresentam esse tipo de sofrimento devem abranger diferentes aspectos desses sujeitos, sendo alguns deles: a comunidade, a organização familiar, as questões psíquicas e os determinantes sociais. Dessa forma, não há como examinar tais questões de forma isolada. Somente através da articulação destes segmentos é que a prevenção primária e o tratamento ao uso prejudicial destas substâncias podem se tornar realmente efetivos (Brasil, 2011).

Nessa perspectiva, considerando a ESF, o papel dos Agentes Comunitários de Saúde (ACS) seria essencial, devido às atribuições estratégicas e complexas, pois são vinculados ao contato direto e conhecimento da comunidade em que trabalham e residem. Estes profissionais representam o elo entre a equipe de saúde e a população, com a função precípua de produzir troca de conhecimentos entre o universo popular e as equipes de saúde, visando com isso, entre outras coisas, à facilitação do acesso dos usuários aos serviços (Nogueira, Silva, \& Ramos, 2000; Vieira \& Tittoni, 2015).

Neste contexto, o ACS assume uma posição bidirecional, enquanto residente da comunidade em que trabalha e integrante da equipe de saúde. Vive, portanto, constituindo-se em ambiguidade cercada de grande complexidade, visto que adentra nos domicílios, recebe as 
queixas da população, não raramente comprometendo-se de forma direta com a necessidade de dar respostas e encaminhamentos aos problemas encontrados. E, por outro lado, deve ajustar-se à equipe e deliberar conforme as possibilidades e os limites dela e do próprio sistema de saúde (Peres, Caldas, Silva, \& Marin, 2011). Especificamente no campo da saúde mental, no que tange às questões relacionadas ao uso de álcool e outras drogas, estas mesmas facilidades tomam dimensões diferentes e precisam ser problematizadas com esses trabalhadores. Sabemos que o imaginário popular vem cheio de assertivas em relação aos usuários de álcool e outras drogas, e que existe uma grande carga de preconceito e ajuizamento de valor que também fazem parte do repertório discursivo da comunidade, o qual se replica no discurso desses trabalhadores, revelando-se, principalmente, pela estigmatização dos usuários de drogas (Melo, Assunção, \& Vecchia, 2016). Tal fato pode dificultar o acesso e o acolhimento dessa demanda nas Unidades Básicas de Saúde. Para Barros, Chagas e Dias (2009), os discursos dos ACS corroboram, explicitamente, com a ideia de distanciamento das pessoas com transtorno mental, já que os profissionais referem dificuldades na aproximação, em virtude do medo causado pelo estranhamento diante de determinados comportamentos.

Logo, torna-se relevante o conhecimento do cotidiano dos ACS, de seus anseios e de suas perspectivas sobre as questões relacionadas ao uso e usuários de álcool e de outras drogas. Assim, potencializa-se a elucidação dos entraves para o efetivo acesso desta clientela às ESF, bem como auxilia-se a criar desfechos terapêuticos possíveis que sejam mais inclusivos a estes usuários no âmbito da Atenção Primária à Saúde (APS).

O objetivo deste estudo foi de compreender o entendimento dos ACS em relação à abordagem da temática das drogas no território em que atuam.

\section{Aspectos Metodológicos}

Trata-se de estudo de natureza qualitativa, realizado no período de março a junho de 2014 , em uma Unidade Básica de Saúde da Família (UBSF) do município de Campo Grande, MS. Esta UBSF fez parte do Programa de Educação pelo Trabalho para a Saúde - Redes de Atenção (PET-Saúde/Redes), centrado no eixo da Rede de Atenção Psicossocial: Enfrentamento do Álcool, Crack e outras Drogas, da Universidade Federal de Mato Grosso do Sul (UFMS).

Os sujeitos da pesquisa foram os 6 ACS desta equipe, que também participavam das atividades do referido projeto. Assim sendo, todos os ACS desta unidade de saúde, envolvidos na realização do PET-Redes da UFMS, foram ouvidos, de modo que o fechamento amostral se deu por exaustão, o que ocorre quando ". . . os participantes foram aqueles que faziam parte do universo definido pelo pesquisador" (Fontanella, Ricas, \& Turato, 2008, p. 22).

A técnica de investigação escolhida foi a entrevista, composta por uma questão norteadora: "Como é o seu trabalho em relação aos membros da comunidade que são usuários de álcool/outras drogas?". Realizaram-se as gravações em áudio, as quais foram transcritas para análise minuciosa. As entrevistas foram realizadas individualmente, em local restrito, para que os participantes tivessem maior liberdade ao expressarem suas contribuições para o estudo.

Os dados foram organizados pela técnica do Discurso do Sujeito Coletivo (DSC) (Lefèvre \& Lefèvre, 2000), cujas etapas de análise foram: leitura flutuante dos depoimentos, destaque 
das expressões-chave (ECH) e das Ideias Centrais (IC) e, por fim, a construção do DSC, redigido na primeira pessoa do singular e composto pelas ECH, que têm a mesma IC.

A pesquisa foi aprovada pelo Comitê de Ética em Pesquisa da UFMS, sob o protocolo 545.606.

\section{Análise dos Resultados e Discussão}

Ter como sujeito do estudo os ACS foi uma atividade desafiadora, tendo em vista que estes representam um misto de profissional da saúde e membro da comunidade. São atores sociais que estão, em todo o tempo, em construção, desconstrução e ressignificação de "verdades" que transitam tanto no campo da produção da saúde (aqui representado pelo serviço de saúde, com suas nuances geralmente normativas) quanto na esfera da vida cotidiana, que é essencialmente complexa e imprevisível, portanto, avessa à normatividade.

No caso dos ACS, isto toma dimensão crítica, posto que, na atenção aos usuários de drogas, estes profissionais se deparam com o conflito entre a influência da percepção leiga e a ciência, as quais podem interferir em suas perspectivas sobre o processo saúde-doença em questão, prejudicando a integralidade do cuidado (Batista \& Souto, 2017). Por outro lado, entre estes profissionais, é comum o relato de que têm dificuldades em estabelecer limites entre sua atuação profissional e a relação pessoal que construíram com os membros do território/comunidade que residem na microárea populacional sob sua responsabilidade (Melo et al., 2016), que é também onde o ACS reside.

Neste momento, discutiremos dois Discursos do Sujeito Coletivo (DSC) que emergiram da análise dos dados, os quais balizarão a problematização da discussão: DSC 1 - A questão do álcool e outras drogas no cotidiano dos ACS; DSC 2 - Usuários de álcool e drogas e a busca por tratamento.

No contexto dos problemas relacionados ao uso abusivo de álcool/outras drogas, os ACS podem contribuir para minimizar as consequências desta realidade, com intervenções preventivas, educativas e mediando ações assistenciais. Nessa perspectiva, os ACS têm como uma de suas atribuições promover a orientação e o aconselhamento das famílias sobre a verdadeira dimensão do abuso do álcool e outras drogas e das suas consequências. Podem, portanto, estimular as pessoas a cuidarem e preservarem a sua saúde e, em caso de necessidade, mediar o acolhimento desses indivíduos nos diferentes pontos de atenção da RAS, incluindo a própria ESF. Esta abordagem deve ser realizada respeitando-se os princípios dos valores humanos e culturais dos cidadãos, entre eles o uso da linguagem correta e acessível no oferecimento de uma informação objetiva, clara, honesta, educativa e eficaz (Castanha \& Araújo, 2006).

Apesar de estas atribuições estarem claras em termos de políticas de saúde, o que se observa, no cotidiano do trabalho, é que as questões relativas ao uso de álcool/drogas que ocorrem na área sob responsabilidade do ACS geralmente são negligenciadas por estes profissionais, por motivos de insegurança e despreparo. Isto contribui para o sentimento de uma dupla vulnerabilidade, pela possibilidade de contato com os mais variados tipos de violência e pelo sentimento de inaptidão para resolução de questões complexas (Melo et al., 2016), características comuns e, até certo ponto, constitutivas ao modo de vida dos usuários de drogas. Estas questões estão presentes no DSC1: 


\section{DSC 1 - A Questão do Álcool e Outras Drogas no Cotidiano dos ACS}

"Isso é assunto de polícia, nós não temos como nos envolver. Eles não gostam que a gente pergunte sobre isso, acham que vamos delatar, ser dedo duro. Eu tenho medo de tocar nesse assunto principalmente com homens, pois você entra na casa sozinha e você não sabe do que ele é capaz, é arriscar a vida da gente. Eu passo aperto, por isso não gosto muito de misturar esses assuntos. O álcool é mais fácil, pois geralmente a familia pede ajuda para a gente. Agora, com esse povo que usa droga mesmo, como o crack e a maconha, eu não me envolvo."

Constata-se no DSC1 que existe um tabu envolvendo esse tema, tanto por parte dos familiares e usuários quanto por parte dos profissionais da saúde. Embora sejam profissionais envolvidos na produção da saúde, os ACS preferem não tratar desse assunto com as famílias, seja por acreditarem que seria uma invasão de privacidade, ou por não sentirem segurança (Waidman, Costa, \& Paiano, 2012; Melo et al., 2016; Batista \& Souto, 2017). Isto pode ser explicado pelo fato de que, apesar do crescimento de políticas públicas nesse campo, o profissional ainda não se considera com competência suficiente, nem enxerga em sua posição a condição de trabalhar essas questões de forma harmoniosa e efetiva junto à comunidade (Rios, 2007; Vallegas \& Souza, 2020).

Além disso, tal constatação revela que, ao relegarem os problemas relacionados ao uso abusivo de álcool e outras drogas ao setor da segurança pública (polícia), os ACS demonstram que, nesse aspecto, não atrelam tais situações ao campo da saúde, nem consideram que a complexidade do problema sofre influências múltiplas e que os determinantes sociais em saúde interferem fortemente no processo saúde-doença. Tal fato contradiz a lógica de trabalho da ESF, ao passo que dificulta, no campo das práticas, a reorientação do modelo assistencial para ações de saúde equânimes e que contemplem a integralidade do cuidado (Oliveira, McCallum, \& Costa, 2010), principalmente se considerarmos que os problemas do território relacionados ao uso de drogas requerem, naturalmente, ações intersetoriais para que sejam adequadamente manejados.

Identificam-se, assim, lacunas na atenção à saúde destes usuários, com limitações importantes dos serviços de saúde sobre demandas reais, as quais se perpetuam como mazelas socialmente cristalizadas, tradicionalmente marcadas pela exclusão e, até certo ponto, pela negação deste problema no território de saúde. Se os ACS, que pertencem à comunidade em que atuam, encontram dificuldades para atenderem às necessidades de saúde dos usuários em relação ao uso de drogas, as barreiras para os demais profissionais da equipe diante de tais demandas são ainda maiores. No cotidiano dos serviços, observam-se investimentos em ações de saúde de baixo impacto, pouco resolutivas e que, por fim, negligenciam necessidades de saúde que interferem no cotidiano da comunidade (Barbosa, Elizeu, \& Penna, 2013). Na mesma perspectiva, distancia-se a transformação do sistema e do próprio processo de trabalho em saúde, em oposição aos princípios do SUS e da ESF (Schneider et al., 2013).

Ao abordarmos as questões relacionadas à atenção à saúde das pessoas com problemas relacionados ao álcool e outras drogas, constatamos que a busca por tratamento por parte desta população ainda enfrenta barreiras. A recusa formal destes trabalhadores em reconhecer o uso de álcool e outras drogas como demanda de sofrimento, logo, objeto de 
atuação da área da saúde, impõe relevante barreira de acesso destes usuários ao sistema de saúde (Melo et al., 2016). Acreditávamos, inicialmente que localizaríamos no discurso dos ACS angústias relacionadas à pouca capacidade técnica em conduzir esses casos, fato este discutido por Campos (2011), que destacou que as demandas de saúde mental na atenção primária são consideradas como grandes e diversificadas pelos próprios trabalhadores. Estas demandas podem, portanto, produzir angústia nas equipes, exigindo um trabalho de compreensão mais amplo, o que tenciona certo tipo de procedimento mecanizado de queixa-conduta, representado principalmente pelos protocolos utilizados em outras áreas de conhecimento, ao passo que, por serem consideradas complexas, as questões acerca desse problema justificam a dificuldade no manejo dos casos. Entretanto, o fato aqui constatado é mais grave do que a angústia dos trabalhadores em não se sentirem preparados em lidar com esta demanda, pois não se alcança nem a possibilidade de reconhecer aquela queixa como uma responsabilidade da sua área de atuação. Isto poderia ser suplantado se os ACS entendessem que as condições sociais e econômicas podem ser norteadoras da construção de estratégias para o enfrentamento das implicações que caracterizam a singularidade de seu trabalho (Vieira \& Tittoni, 2015).

\section{DSC 2 - Usuários de Álcool e Drogas e a Busca por Tratamento}

"Eles (os pacientes) têm medo de se tratar, pois os traficantes retaliam, pois vai ser um freguês a menos. Eu tenho alguns pacientes com problema mental e de drogas na minha área, mas é raro eles virem aqui pedir ajuda. Vocês pedem para a gente mexer com isso, mas depois vão embora para a casa de vocês e quem fica aqui somos nós. Esses dias eu até fiquei surpreso, uma menina na rua me abordou perguntando sobre preventivo e teste rápido de HIV, ela está gestante e é usuária de drogas. Então eu dou atenção pra essa gente quando vêm me procurar, não quero muito contato não. Não vou negar o serviço, mas também não vou procurar por eles não."

Diante disso, reforça-se a constatação de que o acesso ao serviço de saúde, quando efetivado, geralmente é feito pelo próprio paciente, o que vai na contramão dos pressupostos da ESF (Rios, 2007). O discurso mostra que não há, no cotidiano dos serviços de saúde, as ações de vigilância em saúde, busca ativa de sujeitos em situações de risco e vulnerabilidade, tampouco ações para o enfrentamento do problema junto à comunidade e outros setores da sociedade.

Cabe lembrar que, especialmente entre usuários de álcool, crack e outras drogas, a vulnerabilidade é potencializada, sendo necessário apoio não somente ao usuário, mas também aos seus familiares (Luppino et al., 2013). O sucesso das ações preventivas/terapêuticas realizadas nesse sentido está diretamente ligado à amplitude da rede de apoio que estes sujeitos conseguirão estabelecer na sociedade, considerando-se aí os equipamentos sociais, equipamentos de saúde, processo de reinserção (convívio, emprego), entre outros.

A ESF pode funcionar como um catalisador de áreas de prevenção, promoção e recuperação da saúde das pessoas com problemas relacionados ao álcool, assim como a outras drogas, de forma integral e contínua. É necessário investir em áreas que procurem trazer uma formação diversificada e consistente para toda a equipe do ESF, com relação aos 
determinantes do processo saúde/doença (Ronzani \& Silva, 2008), bem como em situações complexas.

Tais achados deixam clara a negação desta problemática no território, sinalizando também uma grave fragilidade nessa rede de cuidado, uma vez que a ESF, nesse estudo representada pelo processo de trabalho do ACS, cujo papel seria de ordenadora das demandas no território, tem assumido um papel declaradamente distante do sofrimento de uma parcela importante da população. Portanto, os participantes deste estudo, ao manterem esse distanciamento de uma demanda real com a qual não se sentem aptos a atuar, evitam o enfrentamento de um situação angustiante, reveladora de temores inerentes à possibilidade de identificação com a fragilidade do outro (Melo et al., 2016).

No entanto, não podemos deixar de problematizar a questão levantada aqui pelos ACS, de que se sentem vulneráveis ao tocar nessas questões de álcool e drogas no seu local de moradia. Estes atores entendem que existem arranjos sociais que não podem ser negligenciados, que passam não exclusivamente pela violência mas que estão relacionados de certa forma a essa temática, implicando uma barreira para sua atuação. Além disso, temos que considerar as dificuldades que os ACS encontram ao abordar o assunto, pois são particularmente afetados emocionalmente, uma vez que se deparam cotidianamente com situações diante das quais sentem-se despreparados e impotentes. Assim como o fato de residirem no território em que atuam pode ser facilitador em uma série de outros aspectos do processo de trabalho, nesse caso específico, é apontado como uma fragilidade para sua atuação. Esta, por sua vez, deveria promover atenção integral aos direitos de saúde do usuário de drogas, preconizada pelo SUS, na medida em que tem potencialidade para estabelecimento de vínculo e da longitudinalidade (Melo et al., 2016), atributos da ESF.

Na perspectiva da Educação Permanente em Saúde direcionada aos ACS (Batista \& Souto, 2017; Vallegas \& Souza, 2020), torna-se necessário discutir formas de abordar, reflexivamente, as questões relacionadas ao uso de álcool e outras drogas e, ao mesmo tempo, não expor o ACS a situações de fragilidade diante da comunidade. Talvez, assim, os ACS se sintam seguros para identificar esta temática como uma demanda de saúde pública e, consequentemente, consigam atuar de forma mais resolutiva junto à comunidade onde atuam.

\section{Conclusões}

A abordagem dos ACS sobre a temática álcool e/ou outras drogas no cotidiano do serviço de saúde é bastante ponderada, cercada de medos e julgamentos, estando a insegurança e inabilidade elencadas como aspectos centrais para as limitações relatadas nos discursos. 0 profissional prefere não abordar o assunto, mesmo que identifique a necessidade, limitando suas intervenções apenas à manifestação voluntária dos usuários, e declara se sentir exposto a situações de violência que cercam esta temática, sentindo-se desprotegido no seu local de moradia, que também faz parte do território em que atua como profissional de saúde.

Para o enfrentamento e a transformação dessa realidade, são necessárias práticas de apoio que possam levar em consideração essas barreiras. Assim, há de se investir em oportunidades para escutar as inseguranças destes trabalhadores, construindo, colaborativamente, estratégias para a modificação do entendimento de que ofertar cuidado para esta população específica pode colocá-los em situação de risco ou vulnerabilidade. 


\section{Referências}

Barbosa, S., Elizeu, T.S., \& Penna, C. M. M. (2013). Ótica dos profissionais de saúde sobre acesso à atenção primária à saúde. Ciência e Saúde Coletiva, 18(8), 2347-2357. doi:https:// doi.org/10.1590/S1413-81232013000800019

Barros, M. M., Chagas, M., \& Dias, M. S. A. (2009). Saberes e práticas do agente comunitário de saúde no universo do transtorno mental. Ciência e Saúde Coletiva, 14(1), 227-232. doi:https://doi.org/10.1590/S1413-81232009000100028

Batista, K., \& Souto, B. G. A. (2017). Perceptions and practices of community health workers in the care of drug users. ABCS Health Sciences, 42(3), 129-136. doi:https://doi.org/10.7322/ abcshs.v42i3.1072

Brasil. Ministério da Saúde (2001). Programa saúde da família. Brasília, DF: Ministério da Saúde. Brasil. Ministério da Saúde (2011). Passo a Passo das Ações do Departamento de Atenção Básica. Brasília, DF: Secretaria de Atenção Básica.

Campos, R. O., Gama, C. A., Ferrer, A. L., Santos, D. V. D., Stefanello, S., Trapé, T. L., \& Porto, K. (2011). Saúde mental na atenção primária à saúde: Estudo avaliativo em uma grande cidade brasileira. Ciência \& Saúde Coletiva, 16(12), 4643-4652. doi:https://dx.doi. org/10.1590/S1413-81232011001300013

Castanha, A. R., \& Araújo, L. (2006). Álcool e agentes comunitários de saúde: Um estudo das representações sociais. Psico-USF, 11(1), 85-94. doi:https://doi.org/10.1590/ S1413-82712006000100010

Fontanella, B., Ricas, J., \& Turato, E. R. (2008). Amostragem por saturação em pesquisas qualitativas em saúde: Contribuições teóricas. Cadernos de Saúde Pública, 24(1), 17-27. doi:https://doi.org/10.1590/S0102-311X2008000100003

Galavote, H. S., Prado, T. N., Maciel, E. L. N., \& Lima, R. C. D. (2011). Desvendando os processos de trabalho do agente comunitário de saúde nos cenários revelados na Estratégia Saúde da Família no município de Vitória (ES, Brasil). Ciência \& Saúde Coletiva, 16(1), 231-240. doi:https://doi.org/10.1590/S1413-81232011000100026

Lefèvre, F., \& Lefèvre, A. M. (2000). O discurso do sujeito coletivo: Uma nova abordagem metodológica em pesquisa qualitativa. Caxias do Sul: EDUCS.

Luppino, J., Nunes, M. C., Silva, M. C., Piquini, M. K, Fornari, P. F., Franco, R. L., . . Gianini, R. J. (2013). Abordagem de usuários de drogas e redução de danos: Atualização do conhecimento de agentes comunitários de saúde em Sorocaba-SP. Revista da Faculdade de Ciências Médicas de Sorocaba, 15(4), 116-120.

Melo, B. C. A., Assunção, J. I. V., \& Vecchia, M. D. (2016). Percepções do Cuidado aos Usuários de Drogas por Agentes Comunitários de Saúde*. Psicologia em Pesquisa, 10(2), 57-66. doi:https://dx.doi.org/10.24879/201600100020060

Merhy, E. E. (2003). O trabalho em saúde: Olhando e experenciando o SUS no cotidiano. São Paulo: Hucitec.

Nogueira, R. P., Silva, F. B., \& Ramos, Z. V. O. (2000). A vinculação institucional de um trabalhador "sui generis": O Agente Comunitário de Saúde. Rio de Janeiro: Cadernos IPEA.

Oliveira, J., Mccallum, C.A., \& Costa, H.(2010). Representações sociais de Agentes Comunitários de Saúde acerca do consumo de drogas. Revista da Escola de Enfermagem da USP, 44(3), 611-618. doi:https://dx.doi.org/10.1590/S0080-62342010000300009 
Peres, C. R. F. B., Caldas, A. L. Júnior, Silva, R. F., \& Marin, M. J. S. (2011). The community health agent and working as a team: The easy and difficultaspects. Revista da Escola de Enfermagem da USP, 45(4), 905-911. doi:https://dx.doi.org/10.1590/S0080-62342011000400016

Rios, I. C. (2007). Rodas de conversa sobre o trabalho na rua: Discutindo saúde mental. Physis: Revista de Saúde Coletiva, 17(2), 251-263. doi:https://dx.doi.org/10.1590/ S0103-73312007000200003

Ronzani, T. M., \& Silva, C. (2008). O Programa Saúde da Família segundo profissionais de saúde, gestores e usuários. Ciência e Saúde Coletiva, 13(1), 23-34. doi:https://dx.doi. org/10.1590/S1413-81232008000100007

Schneider, J. F., Roos, C. M., Olschowsky, A., Pinho, L. B., Camatta, M. W., \& Wetzel, C. (2013). Atendimento a usuários de drogas na perspectiva dos profissionais da estratégia saúde da família. Texto \& Contexto - Enfermagem, 22(3), 654-661. doi:https://dx.doi.org/10.1590/ S0104-07072013000300011

Vallegas, A., Souza, Â., Sanches, L., \& Alves, L. (2020). Permanent health education in the work process of community health workers. Research, Society and Development, 9(4), e129942962. doi:http://dx.doi.org/10.33448/rsd-v9i4.2962

Vieira, L. C. N., \& Tittoni, J. (2015). Entre imagens e palavras: As Agentes Comunitárias de Saúde comoartífices. Pesquisase Práticas Psicossociais, 10(1), 115-128. Recuperadodehttp://pepsic. bvsalud.org/scielo.php?script=sci_arttext\&pid=\$1809-89082015000100010\&lng=pt\&tlng= Waidman, M. A., Costa, B., \& Paiano, M. (2012). Percepções e atuação do Agente Comunitário de Saúde em saúde mental. Revista da Escola de Enfermagem da USP, 46(5), 1170-1177. doi:https://dx.doi.org/10.1590/S0080-62342012000500019

\section{Sobre os autores:}

Ana Carolina Ametlla Guimarães: Mestra em Saúde da Família pela Universidade Federal de Mato Grosso do Sul (UFMS). Graduada em Medicina pela Universidade Federal do Rio de Janeiro (UFRJ). Atualmente, é coordenadora da área de Saúde Mental na Secretaria Municipal de Saúde de Campo Grande, Mato Grosso do Sul. E-mail: carolametlla@hotmail.com, Orcid: http://orcid.org/0000-0001-5473-5385

Iolly Nacasato: Graduada em Nutrição pela Universidade Federal de Mato Grosso do Sul (UFMS). Atualmente, é cirurgiã-dentista. E-mail: iollynacasato@yahoo.com.br, Orcid: http://orcid.org/0000-0002-3322-8656

Rayssa Nacasato: Graduada em Odontologia pela Faculdade de Odontologia da Universidade Federal de Mato Grosso do Sul (UFMS). Atualmente, é nutricionista. E-mail: rayssanacasato@yahoo.com.br, Orcid: http://orcid.org/0000-0003-2843-7514

Leila Foerster Merey: Doutora e mestre em Saúde e Desenvolvimento na Região Centro-Oeste pela Universidade Federal de Mato Grosso do Sul (UFMS). Graduada em Fisioterapia pela Universidade Católica Dom Bosco (UCDB). Atualmente, é professora da UFMS. E-mail: leila.ufms@gmail.com, Orcid: http://orcid.org/0000-0003-2267-0841

Carolina Gomes Carrilho: Mestranda do Programa de Pós-Graduação em Psicologia da Saúde na Universidade Católica Dom Bosco (UCDB). Graduada em Psicologia pela UCDB. Atualmente, é psicóloga. E-mail: carolcarrilho1@hotmail.com, Orcid: http://orcid.org/0000-0001-9833-7645

André Barciela Veras: Doutor em Psiquiatria pela Universidade Federal do Rio de Janeiro (UFRJ). Médico e psiquiatra pela UFRJ. Atualmente, é professor da Universidade Estadual do Mato Grosso do Sul (UEMS). E-mail: barcielaveras@hotmail.com, Orcid: http://orcid.org/0000-0002-4986-7639 
Alessandro Diogo De Carli: Doutor em Ciências da Saúde pela Universidade Federal de Mato Grosso do Sul (UFMS). Graduado em Odontologia pela Universidade Federal de Pelotas (UFPel). Atualmente, é professor da UFMS. E-mail: alessandrodecarli@hotmail.com, Orcid: http://orcid.org/0000-0002-4560-4524

Mara Lisiane de Moraes dos Santos: Doutora em Ciências da Saúde pela Universidade de Brasília (UnB). Mestre em Saúde Coletiva pela Universidade Federal de Mato Grosso do Sul (UFMS). Graduada em Fisioterapia pela Universidade Estadual de Londrina (UEL). Atualmente, é professora da UFMS. E-mail: mara.santos@ufms.br, Orcid: https://orcid.org/0000-0001-6074-0041

Recebido em: 21/08/2019

Última revisão: 04/05/2020

Aceite final: 09/06/2020 